\title{
Zukunftsfaktor Mitbestimmung
}

\author{
Marie Seyboth \\ Rainald Thannisch
}

Die Mitbestimmung basiert auf demokratietheoretischen, sozialpolitischen und ethischen Überlegungen. Im Mittelpunkt der wirtschaftsdemokratischen Begründung steht der Gedanke, dass alle am Wirtschaftsprozess Beteiligten in gleicher Weise an den Entscheidungen teilnehmen sollen. Es geht um die demokratische Kontrolle wirtschaftlicher Macht.

Weiterhin wird insbesondere aus industriesoziologischen Zusammenhängen argumentiert, dass die Mitbestimmung der deutschen Wirtschaft geholfen habe, sich in schwierigen Weltmärkten für hoch diversifizierte Qualitätsprodukte dauerhaft zu behaupten. So betont der Bericht der gemeinsamen Kommission Mitbestimmung von Bertelsmann Stiftung und Hans-Böckler-Stiftung (1998) den Beitrag der Mitbestimmung $\mathrm{zu}$ einer produktiven Überbrückung von Hierarchien im Betrieb sowie zu einer demokratischen Integration der Gesellschaft insgesamt.

Diese Argumente wiegen schwer und werden auch von den Gegnern der Mitbestimmung nicht offen kritisiert. Stattdessen verweisen sie darauf, dass der Wert der Mitbestimmung in der globalisierten Welt von heute durch viele Nachteile konterkariert werde.

Den vorläufigen Schlusspunkt dieser insbesondere zwischen 2004 und $2006 \mathrm{sehr}$ prominent geführten Debatte markiert der Bericht der wissenschaftlichen Mitglieder der Regierungskommission zur Modernisierung der deutschen Unternehmensmitbestimmung (Biedenkopf II-Kommission). Sie sehen keinen Grund dafür, der Bundesregierung eine grundlegende Revision der deutschen Unternehmensmitbestimmung vorzuschlagen. Sie führen aus, dass der mitbestimmte Aufsichtsrat einer günstigen Bewertung des deutschen Standorts nicht im Wege stehe und dass es keine Anzeichen dafür gebe, dass mitbestimmte Unternehmen sich unter den veränderten Wirtschaftsbedingungen der Gegenwart und des kommenden Jahrzehnts weniger erfolgreich behaupten werden als in den drei Jahrzehnten seit Verabschiedung des Mitbestimmungsgesetzes im Jahr 1976. ${ }^{1}$
Trotzdem beobachten wir, dass die Angriffe auf die Unternehmensmitbestimmung fernab der großen Schlagzeilen und Tickermeldungen nicht aufgehört haben. Im Gegenteil haben wirtschaftsliberale Wissenschaftler und Institutionen wie beispielsweise der Kronberger Kreis der Stiftung Marktwirtschaft oder das Institut der deutschen Wirtschaft (IW) ihre Kritik an der Unternehmensmitbestimmung wiederholt bekräftigt. Getreu dem Motto „Das stete Wasser höhlt den Stein" werden die teilweise sehr einseitigen Ausführungen zur Mitbestimmung laufend wiederholt. Kern der Aussage ist dabei immer die angebliche Inkompatibilität der Mitbestimmung mit europäischem Recht sowie der aus ihr vermeintlich resultierende Standortnachteil.

Die Debatte hat sich jedoch von der großen politischen Ebene auf die wissenschaftliche und verbandliche Ebene verlagert, wo sie womöglich darauf wartet, von einer zukünftigen Bundesregierung unter Beteiligung der nach wie vor mitbestimmungskritischen FDP wachgeküsst zu werden.

Das ist - wie wir finden - Grund genug, einen kritischen Überblick über aktuelle oder vorgebliche Herausforderungen der Unternehmensmitbestimmung zu geben (Abschnitt 2). Dabei beschränken wir uns auf die prägnantesten Aspekte der aktuellen juristischen und ökonomischen Diskussion. Anschließend stellen wir einige der wichtigsten gewerkschaftlichen Vorschläge für eine Weiterentwicklung der Unternehmensmitbestimmung vor (Abschnitt 3).

\section{AKTUELLE HERAUSFORDERUNGEN FÜR DIE MITBESTIMMUNG}

\section{EUROPÄISIERUNG DES GESELLSCHAFTSRECHTS}

Die Unternehmensmitbestimmung ist in Europa keineswegs isoliert. 19 von $27 \mathrm{EU}$ Staaten kennen eine Arbeitnehmerpartizipation im obersten Unternehmensorgan. Die Mitbestimmung ist als Prinzip der Arbeitnehmerpartizipation in Europa aner- kannt und verwirklicht einen legitimen Teilhabeanspruch der Arbeitnehmerinnen und Arbeitnehmer. Eine Einschränkung der Partizipation würde den Zielvorstellungen des EG Vertrags und dem Konzept der europäischen Sozialpolitik zuwider laufen.

Vor dem Hintergrund unterschiedlicher Ansätze der Arbeitnehmerbeteiligung in Europa haben sich die Mitgliedstaaten für den Weg der Vereinbarung entschieden, um die Rolle der Arbeitnehmer/innen und Gewerkschaften in Unternehmen europäischer Rechtsform zu sichern.

So sieht die Richtlinie über die Arbeitnehmerbeteiligung in der Europäischen Aktiengesellschaft (SE) ${ }^{2}$ vor, dass die Mitbestimmung in Verhandlungen zwischen der Unternehmensleitung und einem besonderen Verhandlungsgremium der Arbeitnehmerinnen und Arbeitnehmer (unter Einbeziehung von Gewerkschaften) festgelegt wird. Sollte es in diesen Verhandlungen zu keiner Einigung kommen, greifen gesetzliche Auffangregelungen, die sich tendenziell am weitestgehenden Niveau der Mitbestimmung in den vormaligen Rechtsordnungen orientieren. Der deutsche Gesetzgeber hat die SE-Richtlinie Ende 2004 in nationales Recht umgesetzt.

\footnotetext{
1 Vgl. Kommission zur Modernisierung der deutschen Unternehmensmitbestimmung (2006), Bericht der wissenschaftlichen Mitglieder der Kommission, download: http://www.dgb.de/themen/ mitbestimmung/untern_mitbest/weiterentwicklung. htm.

2 Richtlinie 2001/86/EG des Rates vom 8. Oktober 2001 zur Ergänzung des Statuts der Europäischen Gesellschaft hinsichtlich der Beteiligung der Arbeitnehmer.
}

\footnotetext{
Marie Seyboth, Justitiarin und Bereichsleiterin Mitbestimmung und Unternehmenspolitik beim DGB Bundesvorstand, Berlin. e-mail: marie.seyboth@dgb.de Rainald Thannisch, Diplom-Volkswirt, politischer Referent im Bereich Mitbestimmung und Unternehmenspolitik beim DGB Bundesvorstand, Berlin. e-mail: rainald.thannisch@dgb.de
} 
Für die Arbeitnehmerbeteiligung bei einer grenzüberschreitenden Verschmelzung von Unternehmen gelten vergleichbare Regeln. Hier können sich jedoch die Geschäftsleitungen der betroffenen Unternehmen dazu entschließen, auf Verhandlungen zur Arbeitnehmerbeteiligung zu verzichten und gleich die gesetzlichen Auffangregelungen anwenden. Das entsprechende Gesetz trat Ende 2006 in Kraft.

Die Gewerkschaften haben derzeit mehr praktische Erfahrungen mit der SE, so dass sich die folgenden Ausführungen auf diese beziehen. Nach aktuellen Angaben der Hans-Böckler-Stiftung (Stand: Juni 2008) gibt es in der EU 43 operativ tätige SE, davon 19 in Deutschland. ${ }^{3}$

In den bislang bekannten Verhandlungsergebnissen zur Mitbestimmung in großen Unternehmen ehemals deutscher Rechtsform (z. B. Fresenius SE, Allianz SE, BASF SE) zeigt sich, dass die quasi-paritätische Mitbestimmung und die Einbeziehung von Gewerkschaftsvertreter/innen im obersten Unternehmensorgan beibehalten wird. Bezüglich der Rechte des SE-Betriebsrats und seiner Ressourcenausstattung konnten teilweise sogar über die Auffanglösung hinausgehende Regelungen erreicht werden. In einer ersten Bilanz der SE-Regelungen heben die Gewerkschaften als weiteres positives Ergebnis hervor, dass durch die Mitbestimmung in der SE Arbeitnehmer/innen in Ländern ohne nationale gesetzliche Mitbestimmungsregelungen in den Schutzbereich der Unternehmensmitbestimmung gelangen. Aus der Sicht der Gewerkschaften negativ ist jedoch die Möglichkeit des sogenannten Einfrierens von Mitbestimmungsrechten, d. h. die Mitbestimmung, die in der Gründungsphase ausgehandelt wird, bleibt bestehen, auch wenn sich die Unternehmensgröße signifikant ändert. Dieser Missstand muss bei der demnächst anstehenden Revision der SERichtlinie behoben werden, zumal das Einfrieren Unternehmen als Strategie empfohlen wird, um sich vor der Mitbestimmung zu „schützen“. Dies widerspricht eindeutig dem Geist der Richtlinie. Denn das Grünbuch der Kommission betont bereits 1975, "dass es in wachsendem Maße als ein Gebot der Demokratie erkannt werde", die Arbeitnehmer/innen in die sie betreffenden Unternehmensentscheidungen einzubeziehen (Bulletin der EG Beilage 4/25, S. 12).

Eine andere viel diskutierte These befasst sich damit, ob aufgrund der neuesten EuGH Rechtsprechung zur Niederlas- sungsfreiheit ausländische Kapitalgesellschaften, die (ausschließlich) in Deutschland tätig sind, zum einen nach geltendem Recht der deutschen Unternehmensmitbestimmung unterliegen, oder ob es zum anderen europakonform ist, auf diese Gesellschaften die deutsche Unternehmensmitbestimmung zu erstrecken. Aus gewerkschaftlicher Sicht ist beiden Thesen zuzustimmen.

Nach derzeitigem Recht unterliegen alle Kapitalgesellschaften, die ihren tatsächlichen Sitz in Deutschland haben, der deutschen Unternehmensmitbestimmung. Zwar sind in $\$ 1$ MitbestG 76 die ausländischen Rechtsformen nicht aufgeführt, dies ist aber allein damit zu begründen, dass es im Jahr 1976 das Phänomen der Auslandsgesellschaften mit Sitz in Deutschland nicht gab. Diese Gesellschaften galten in Deutschland, bis zur Rechtsprechung des EuGH zur Niederlassungsfreiheit, als nicht rechtsfähig. Ihnen standen vielmehr nur inländische Rechtsformen zur Verfügung und somit galten auch wiederum die Voraussetzungen des Mitbestimmungsgesetzes 1976.

Der Wille des Gesetzgebers war also, Arbeitnehmerinnen und Arbeitnehmer sowie die Gewerkschaften im Aufsichtsrat von Kapitalgesellschaften ab einer bestimmten Größe zu beteiligen. Deshalb ist eine analoge Anwendung des Mitbestimmungsgesetzes auf Auslandsgesellschaften mit Verwaltungssitz im Inland folgerichtig und verstößt auch nicht gegen europäisches Recht. ${ }^{4}$

Die wissenschaftlichen Mitglieder der Biedenkopf II-Kommission haben zwar von einer Empfehlung zur Erstreckung des deutschen Mitbestimmungsrechts auf ausländische Kapitalgesellschaften mit inländischem Verwaltungssitz abgesehen, da nach ihrer Ansicht zunächst die zukünftige quantitative Entwicklung abgewartet werden sollte, verweisen jedoch darauf, „dass der deutsche Gesetzgeber gemeinschaftsrechtlich nicht daran gehindert ist, solche Unternehmen jedenfalls dann der Mitbestimmung zu unterwerfen, wenn sich ihre betriebliche Organisation einschließlich der Arbeitnehmer im Wesentlichen im Inland befindet und diese Arbeitnehmer nicht nach dem Recht des Gründungsstaates ein Mitbestimmungsrecht haben".

Auch Walter Bayer vertritt in seinem Rechtsgutachten die Auffassung, dass die deutsche Unternehmensmitbestimmung nicht gegen europäisches Recht verstößt. Die Erstreckung auf Auslandsgesellschaf- ten stelle zwar eine Beschränkung der Niederlassungsfreiheit dar, diese sei jedoch durch ein zwingendes Allgemeininteresse gerechtfertigt. ${ }^{5}$

Letztendlich muss das Europaparlament hier Position zugunsten der Unternehmensmitbestimmung beziehen, wenn das Versprechen eines sozialen Europas nicht nur eine Worthülse sein soll.

\section{FINANZMARKTORIENTIERUNG DER WIRTSCHAFT}

Zunächst einmal gilt es, die bei den Wirtschaftsverbänden und Teilen der Wirtschaftspresse beliebten Vorurteile bezüglich der ökonomischen Wirkung der Unternehmensmitbestimmung zu widerlegen: So führen Kai Kühne und Dieter Sadowski in einer aktuellen Arbeit zur Diskrepanz zwischen dem massenmedialen Diskurs zur Mitbestimmung und den Ergebnissen der empirischen Wirtschaftsund Sozialforschung aus: „Gemäß dem aktuellen Stand des empirischen Wissens muss [...] davon ausgegangen werden, dass Mitbestimmung nicht zu messbaren Effizienzeinbußen führt; aus ökonomischer Sicht ist die Regelung insofern unproblematisch".6 Einige neue Studien schreiben der Unternehmensmitbestimmung sogar wirtschaftlich vorteilhafte Effekte zu. So kann Simon Renaud in einer aktuellen Studie einen - über die bereits bekannten positiven Produktivitätseffekte hinausgehenden - positiven Einfluss der quasi-paritätischen Unternehmensmitbestimmung auf das Geschäftsergebnis nach Steuern ermitteln. ${ }^{7}$

3 Weiterführende Informationen unter www.workerparticipation.eu.

4 Vgl. ausführlich Seyboth, M. (2008): Die Mitbestimmung im Licht der beabsichtigten Neuregelung des Internationalen Gesellschaftsrecht; in: Arbeit und Recht 2, S. 132-136.

5 Bayer, W. (2004), Die Rechtsprechung des Europäischen Gerichtshofes zur Niederlassungsfreiheit und die deutsche Unternehmensmitbestimmung, Rechtsgutachten im Auftrag der Hans-BöcklerStiftung, S. 32.

6 Kühne, K./Sadowski, D. (2008): Empirische Mitbestimmungsforschung und Öffentlichkeit, IAAEG Discussion Paper Series 2008/01. Vgl. für weitere Literaturangaben auch Thannisch, R. (2007): Ein Meilenstein für die Mitbestimmungsdebatte, in: Sozialer Fortschritt 6, S. 163-171.

7 Renaud, S. (2007): Dynamic Efficiency of Supervisory Bord Codetermination in Germany, LABOUR 21, S. 689ff. 
Das win/win-Potenzial der Mitbestimmung wird jedoch im Zuge der Finanzmarktorientierung unserer Wirtschaft und den dadurch veränderten Handlungsstrategien im Unternehmen zunehmend in Frage gestellt. Beispielsweise dadurch, dass Manager sich heute oftmals ausschließlich als Agenten der Kapitaleigner verstehen und bestrebt sind, vor allem den Nutzen der Kapitaleigner, neudeutsch Shareholder Value, zu maximieren.

So besteht die große Gefahr, dass diejenigen Anspruchsgruppen an ein Unternehmen systematisch benachteiligt werden, die zur Steigerung des Shareholder Value keinen direkten oder einen vermeintlich zu geringen Beitrag leisten können. Der zunehmende Einsatz von Leiharbeitnehmer/ innen bei weniger qualifizierten Tätigkeiten und deren im Vergleich oftmals sehr viel schlechteres Einkommen und ihre überproportionale Unfallhäufigkeit und Gesundheitsgefährdung weisen in diese Richtung - und geben einen Ausblick auf die verteilungspolitischen und gesellschaftspolitischen Folgen dieser Strategie.

Auch für die Unternehmen ist diese Entwicklung negativ. Wir wissen aus vielen Beispielen, dass der Shareholder-ValueAnsatz das Wachstumspotenzial und die Zukunftsfähigkeit eines Unternehmens gefährdet, weil Investitionen und Innovationen systematisch vernachlässigt werden. Dieser Effekt wird durch den Bedeutungsgewinn von Finanzinvestoren unterstützt, die zu den entschlossensten Vertretern des Shareholder-Value-Ansatzes zählen. Insbesondere die Geschäftspolitik von Private Equity Gesellschaften und Hedge Fonds ist gekennzeichnet durch die in Extremfällen bis an den Rand der Leistungsfähigkeit gehende Überschuldung der gekauften Unternehmensteile, sowie durch die radikale Ausnutzung so genannter "Wirtschaftlichkeitsreserven". Hier entsteht auch ein sehr hohes Risiko für die Beschäftigten z. B. durch Stellenabbau, durch Lohnverzicht oder durch den Verzicht auf Pensions- bzw. Betriebsrentenzusagen.

Im Gegensatz dazu versuchen Finanzinvestoren oftmals, das Management der von ihnen übernommenen Unternehmen durch finanzielle Anreize auf ihre Unternehmensstrategie zu verpflichten. ${ }^{8}$ Umso wichtiger wird es für die Arbeitnehmervertreter im Aufsichtsrat, nicht nur der Höhe, sondern auch der Zusammensetzung der Vorstandsvergütung und der damit intendierten Lenkungswirkung ihre Aufmerk- samkeit zu schenken. Hier fehlt es derzeit jedoch an einer klaren gesetzlichen Definition der Angemessenheit der Vorstandsvergütung.

Dennoch: Die unbestrittene Kompetenz des mitbestimmten Aufsichtsrates in Vergütungsfragen verdeutlicht, dass die Mitbestimmung im modernen Finanzmarktkapitalismus nicht ohne Einfluss ist. Schon jetzt zeigt sich, dass eine gewerkschaftliche Präsenz im mitbestimmten Aufsichtsrat eine dämpfende Wirkung auf hohe Vorstandsvergütungen sowie auf den Anteil der aktienorientierten Vergütung hat. ${ }^{9}$

Außerdem können Arbeitnehmervertreter/innen im Aufsichtsrat dem Diktat des Shareholder Value widersprechen und die Diskussion um Produkte, Technologien, Märkte und Menschen im Aufsichtsrat gleichberechtigt zur Finanz- und Rentabilitätsdiskussion führen. Dass dies möglich ist, zeigen Arbeitnehmervertretungen tagtäglich in ihrer Arbeit, indem sie die Interessen von Beschäftigten und anderen Anspruchsgruppen erfolgreich und zum Wohl des Unternehmens in die Aufsichtsratsarbeit einbringen. ${ }^{10}$ Eine diesbezügliche fundierte und praxisnahe Handlungshilfe hat erst jüngst die IG Metall vorgelegt. ${ }^{11}$

Selbst wenn es um Finanzinvestoren geht, gibt es Erfolgsmeldungen der Unternehmensmitbestimmung: So können sich Arbeitnehmervertreter im Aufsichtsrat dafür einsetzen, dass beim Verkauf eines Unternehmensteils ein Käufer mit einer langfristigen und nachhaltigen Zielsetzung ausgewählt wird. Best Practice für ein solches Vorgehen ist der Verkauf der ehemaligen Gabelstaplersparte Kion des Linde Konzerns an den Finanzinvestor KKR. Hier waren Arbeitnehmervertreter im Aufsichtsrat maßgeblich in die Verkaufsentscheidung eingebunden. Auch in den von einem Finanzinvestor übernommenen Unternehmen gibt es Einflussmöglichkeiten der Unternehmensmitbestimmung. So ist aus Unternehmensbeispielen bekannt, wie eng die Handlungsspielräume von Geschäftsführungen in Unternehmen im Besitz eines Finanzinvestors bisweilen sind. Nur durch die Mitbestimmung im Aufsichtsrat ist es möglich, die berechtigten Interessen der Beschäftigten und damit auch wichtiges betriebsspezifisches Wissen und Knowhow an den neuen Eigentümer weiterzugeben und mit diesem auf gleicher Augenhöhe in eine Diskussion einzutreten.

Es zeigt sich, dass die Mitbestimmung insbesondere in ihrer Schutzfunktion für die Arbeitnehmer und als Korrektiv zum schrankenlosen Shareholder Value an Bedeutung gewinnt. Diesen wichtigen Beitrag der Mitbestimmung in Zeiten des Finanzmarktkapitalismus unterstreichen auch die wissenschaftlichen Mitglieder der Biedenkopf II-Kommission die zu Recht ausführen:

"Zweifellos lassen sich die Folgen entgrenzter Finanzmärkte nicht ohne weit reichende wirtschafts- und finanzpolitische Maßnahmen überwinden. Aber dessen ungeachtet erscheint es nicht ausgeschlossen, dass sich auch im Rahmen der Mitbestimmung der Arbeitnehmer neue Formen der Begrenzung und damit Stabilisierung des Unternehmensverbandes entwickeln lassen. Der Mitbestimmung im Unternehmen könnten auf diese Weise zusätzliche Aufgaben erwachsen, die dem Unternehmensverband als Ganzem dienen."

\section{POSITION DER GEWERKSCHAFTEN}

Wie gezeigt werden konnte, wird die Mitbestimmung im zunehmenden Finanzmarktkapitalismus immer wichtiger. Um den Herausforderungen auf gleicher Augenhöhe begegnen zu können, bedarf es aus Sicht der Gewerkschaften ${ }^{12}$ einer Wei-

8 Vgl. für den gesamten Abschnitt: Kamp, L. (2007): Zum Einfluss von Private Equity- und HedgeFonds auf die Wirtschaft, in: WSI-Mitteilungen 11, S. 596-603; Kamp, L./Krieger, A. (2005): Die Aktivitäten von Finanzinvestoren in Deutschland, Arbeitspapier 103 der Hans-Böckler-Stiftung.

9 Vgl. Vitols, S. (2008): Beteiligung der Arbeitnehmervertreter in Aufsichtsratsausschüssen: Auswirkungen auf Unternehmensperformanz und Vorstandsvergütung, Studie im Auftrag der HansBöckler-Stiftung, download: www.boeckler.de.

$10 \mathrm{Vgl}$. eine Auflistung von Beispielen unter: http:// www.dgb.de/themen/mitbestimmung/untern_ mitbest/positive_beispiele/index_html.

11 Vgl. IG Metall (2007): Unternehmensstrategie und Innovation, Arbeitshilfen und Organisationshilfen für Aufsichtsräte A1.

12 Vgl. für die gewerkschaftliche Positionsbestimmung: DGB (2006): Mitbestimmung und Teilhabe - Mitbestimmung für die Zukunft, Beschluss des 18. ordentlichen DGB Bundeskongresses, download: www.dgb.de; Hexel, D. (2006): Zum Standort der Unternehmensmitbestimmung heute, in: Verhandlungen des 66. Deutschen Juristentages, Band II/1, S. M49-M75; Stellungnahme der Vertreter der Arbeitnehmer in der Biedenkopf II-Kommission, vgl. Fn. 1; DGB (2008): DGB Vorschläge für ein gesetzliches Maßnahmebündel zur Regulierung der Vorstandsvergütung, Beschluss des DGB Bundesvorstandes vom 3. Juni 2008, download: www.dgb.de. 
terentwicklung der Mitbestimmung und einer Präzisierung des Unternehmensinteresses sowie der Angemessenheit der Vorstandsvergütung im Aktienrecht. Einige der zentralen gewerkschaftlichen Forderungen sollen daher kurz dargestellt werden.

\section{(1) Gesetzlicher Mindestkatalog für zustimmungspflichtige Geschäfte}

Es ist das Ziel der Gewerkschaften, die Mitbestimmung an zentralen unternehmerischen Entscheidungen sicherzustellen. Die Wahrnehmung dieser Aufgaben setzt einen Katalog zustimmungspflichtiger Geschäfte voraus. In einen solchen Katalog gehören alle Maßnahmen der strategischen Ausrichtung des Unternehmens, darunter Betriebsschließungen, Standortverlagerungen und Unternehmensverkäufe; er ist gesetzlich festzuschreiben. Denn die Praxis zeigt, dass die Zustimmungskataloge der Aufsichtsräte häufig zu knapp ausgestaltet sind.

\section{(2) Mitbestimmung in Gesellschaften ausländischer Rechtsform}

Aus Gründen der Rechtssicherheit sollte gesetzlich klargestellt werden, dass ausländische Gesellschaften in die deutsche Unternehmensmitbestimmung einzubeziehen sind. Auch die Empirie spricht für einen gesetzgeberischen Handlungsbedarf: Nach einer aktuellen empirischen Studie der Hans-Böckler-Stiftung finden derzeit in 29 Unternehmen ausländischer Rechtsform mit Tätigkeit in Deutschland die gesetzlichen Regeln zur Mitbestimmung keine Anwendung - eine Zunahme um 12 Fälle seit 2006. Nach wie vor nutzt jedoch keine Kapitalgesellschaft ausländischer Rechtsform Deutschland für einen Verwaltungssitz in mitbestimmungsrelevanter Größe. ${ }^{13}$

\section{(3) Präzisierung der Angemessenheit der Vorstandsvergütung}

Das Aktienrecht gibt „ein angemessenes Verhältnis zu den Aufgaben des Vorstandsmitglieds und zur Lage der Gesellschaft" vor. Dieser Begriff ist jedoch leider sehr unscharf. $\$ 87$ Abs 1 AktG ist daher um den folgenden Absatz zu erweitern:

„Bei der Angemessenheit sind die wirtschaftliche Lage, der Erfolg und die $\mathrm{Zu}$ kunftsaussichten des Unternehmens, die persönliche Leistung des jeweiligen Vorstandsmitglieds sowie des gesamten Vorstands, die Entwicklung der Vorstandsvergütung sowie der Tariflöhne in vergleichbaren Branchen und Unternehmen in Deutschland und Europa zu berücksichtigen. Schließlich gilt es bei der Bemessung die soziale, gesellschaftliche und ökologische Verantwortung des Vorstands und des jeweiligen Vorstandsmitglieds heranzuziehen."

\section{(4) Konkretisierung des Unternehmens- interesses}

Der Vorstand hat bei der Leitung des Unternehmens die Belange der Aktionäre, der Beschäftigten sowie der Allgemeinheit zu berücksichtigen (Unternehmensinteresse). In jüngster Zeit mehren sich jedoch die Stimmen, die sich gegen die interessenplu- ralistische Zielkonzeption wenden und sich für einen Vorrang der Aktionärsinteressen aussprechen. Diese Auffassung knüpft an den Shareholder-Value-Ansatz an. Deshalb ist es den Gewerkschaften ein Anliegen, $\S 76$ Abs. 1 AktG um eine präzise Definition des Unternehmensinteresses zu ergänzen.

\section{FAZIT}

Die Mitbestimmung ist ein soziales und wirtschaftliches Erfolgsmodell. Sie ist als Prinzip der Arbeitnehmerpartizipation in Europa anerkannt und verwirklicht einen legitimen Teilhabeanspruch der Arbeitnehmerinnen und Arbeitnehmer. Die wiederholt seitens der Wirtschaftsverbände geforderte „Anpassung der Mitbestimmung an Europa" ist hingegen eine nur wenig verklausulierte Forderung nach einem Abbau von demokratischen Teilhaberechten. Gleichzeitig wird die Mitbestimmung als Korrektiv eines entfesselten ShareholderValue-Kapitalismus nachgerade unverzichtbar. Daher ist es politisch geboten, diese wichtige Funktion durch eine gesetzliche Weiterentwicklung der Unternehmensmitbestimmung abzusichern. Dies gilt insbesondere für die Erstreckung der deutschen Mitbestimmung auf in Deutschland tätige Unternehmen ausländischer Rechtsform und für die Einführung eines gesetzlichen Mindestkataloges zustimmungspflichtiger Geschäfte.

\footnotetext{
13 Sick, S. (2008): Mitbestimmungsrelevante Unternehmen mit ausländischer/kombiniert ausländischen Rechtsformen, download: www.boeckler.de
} 